\title{
Systematic Review of Chimpanzee Use in Monoclonal Antibody Research and Drug Development: 1981-2010
}

\author{
Raija H. Bettauer
}

Bettauer BioMed Research, McLean, VA, USA

\begin{abstract}
Summary
This survey examines the extent to which live chimpanzees have been used in monoclonal antibody $(\operatorname{mAb})$ research and the drug approval process. The survey covers 193 scientific articles published during the years 1981-2010, as well as preclinical studies leading to the approval of mAb drugs by the Food and Drug Administration of the United States. The frequency of the articles has decreased by more than twothirds from their highs in the late 1980's, and the aggregate number of chimpanzees used in these studies has decreased by more than $90 \%$.

The experimental protocols ranged from single or multiple blood draws to extraction of body fluids and tissue samples, and to multiple, repeated organ biopsies. Many studies involved infecting the chimpanzee(s) with pathogenic organisms and immunization and infusion protocols. Addressing the health history and status of the chimpanzees was an exception rather than the rule, and anesthesia and analgesia were mentioned only in a small minority of the surveyed articles.

In the past two decades, the FDA has approved $32 \mathrm{mAb}$ drugs, but only three of those drugs could be determined to involve the chimpanzee in the preclinical stage. Two of those three drugs have been withdrawn from the market due to their severe adverse effects in human patients. Available alternatives, together with ethical and economic reasons, suggest that the use of the chimpanzee in this manner may not be necessary or appropriate.
\end{abstract}

Keywords: Chimpanzee, monoclonal antibodies, preclinical studies, animal model

\section{Introduction}

Since their discovery in the 1970's, monoclonal antibodies (mAbs) have rapidly become an important tool in life sciences research and one of the most fast-growing products of the pharmaceutical industry. More than $30 \mathrm{mAb}$ drugs have been approved to date by the U.S. Food and Drug Administration (FDA), and hundreds of mAbs have been tested in clinical trials, particularly in the fields of immunotherapy and oncology (Strohl, 2009).

The key characteristic of a mAb is its ability to bind to a specific molecular target, based on the chemical and conformational complementarity of the mAb and the target (Brekke and Sandlie, 2003). Although mAbs initially might have been thought of as a high-technology version of natural antibodies (Ig), to be used in therapy like serum and polyclonal antibodies, their special properties soon gave rise to new applications. The therapeutic targets of mAbs now consist largely of receptors and soluble ligands, and a mAb can be used as an "address label" to deliver a toxin or another drug to the appropriate molecule (Alley et al.,
2009). In cancer therapy, for example, mAbs can be conjugated with toxins or radionuclides to treat or image tumor cells.

The unique characteristics of mAbs influence preclinical studies of $\mathrm{mAb}$ drugs, including the selection of an animal model. As with other drugs, pharmacokinetics and -dynamics (PK \& PD) studies are a critical part of preclinical studies. With mAbs, however, PK \& PD studies typically are more complex than those for small-molecule drugs. For example, because of their large molecular size, mAbs are not eliminated renally for the most part but, rather, through secretion or catabolism, depending on the type of mAb (Wang et al., 2008). Species-specific affinity of a mAb for relevant epitopes, in turn, may extensively affect its elimination rate (Loisel et al., 2007). Immunogenicity also has received attention, as increasingly humanized mAbs may prove immunogenic in animals used in preclinical studies (Wang et al., 2008).

Some primate researchers assert the importance of the chimpanzee model in the development of mAb drugs. While detailed scientific justifications of that argument have not been set forth, the main gist of the argument appears to be the chim-

Received February 10, 2011; accepted in revised form March 09, 2011. 
panzee's phylogenetic proximity to humans and the human immune system (VandeBerg et al., 2006). Moreover, the argument notes that many pharmacological mAb studies already use the chimpanzee. Are chimpanzees, then, truly the animal model of choice in current pharmacological mAb studies - and for future studies as well?

This article sets forth, first, a comprehensive and systematic review of three decades of published peer-reviewed scientific literature that deals with the use of the chimpanzee in $\mathrm{mAb}$ research. The analysis of these scientific studies includes, in addition to their frequency, types of research involved, numbers of chimpanzees used, both in the aggregate and in individual studies, and procedures used on chimpanzees.

Second, this article goes on to review the approval of $\mathrm{mAb}$ drugs by the FDA to ascertain to what extent the chimpanzee has been used as a preclinical animal model in this context. Finally, the discussion section addresses prospective uses of the chimpanzee in $\mathrm{mAb}$ research, including whether such use is necessary or appropriate.

\section{Methods}

\subsection{Survey of scientific articles}

Scope

PubMed is a comprehensive search and retrieval engine of scientific literature maintained by the U.S. National Library of Medicine (The National Institutes of Health, PubMed, http:// www.ncbi.nlm.nih.gov/sites/entrez). To identify articles that deal with the use of the chimpanzee in mAb research, PubMed was searched with "chimpanzee AND monoclonal AND antibody," limiting the search to articles in the English, German, and French languages. This left out three articles in the Japanese language and one in Russian. The first entry in the results dated from December 1981. Consequently, the date range for the survey was defined as the period of Jan.1, 1981 through Dec. 31, 2010. This search produced 274 results.

Next, it was necessary to identify, among the 274 articles, those that did not deal with original research or that did not actually use live chimpanzee(s). As a result of this initial review, 20 articles were found to consist of reviews, opinion pieces, or commentaries. Another 26 articles, while dealing with original research, did not actually use the chimpanzee; they merely mentioned it, usually by reference to another research project. Yet another 18 articles involved an analysis of biological or genetic properties of the chimpanzee, but the sample(s) in question had been retrieved from a genetic data bank, such as GenBank, or the study used a cell line provided by another laboratory. Four articles dealt with research performed on tissues or organs removed from necropsied chimpanzees, and since these articles did not involve live chimpanzees, they were omitted from subsequent, more detailed analyses. Finally, 13 articles - all dating from before 1990 - could not be located for further analysis.

The elimination of the above sub-categories of articles left 193 articles that involved both mAb research and the use of the live chimpanzee. These 193 articles form the base for this sur- vey. It should be pointed out, however, that this article base is likely to underestimate the extent of the use of the chimpanzee in this segment of scientific research. For example, private companies and laboratories do not publish most of the results of their research for proprietary reasons; governmental research may be kept confidential for various reasons; and many other projects are not written up for publication because the project or the results or logistical problems may make it undesirable or unfeasible. Nevertheless, it appears likely that this survey, even if not all-inclusive, is representative of the practices and trends in this area and, as such, provides useful information.

Trends

Overall trends regarding the use of the chimpanzee in $\mathrm{mAb}$ research may be of broader interest for a number of reasons, such as determining possible shifts in the animal models used by the research community. This survey period consisted of three decades, which suggested interrogating relevant trends either by each of the three decades or by 5 -year periods. In the end, the 5-year periods were selected, because this would yield more data points and, therefore, more detail.

\section{Ambiguities}

As discussed in more detail later, there were frequent and substantial gaps in the data in the articles. Ambiguities were particularly frequent in ascertaining the numbers of chimpanzees. Many articles did not state the number of chimpanzees at all. Some others used the singular in describing the chimpanzee use, but this might mean that only one chimpanzee was used or it might signal the species in general. The plural form of the chimpanzee might mean two or any higher number. Therefore, it seemed best simply to base the chimpanzee count on articles where a specific number could be discerned. In this respect, if the data tables or figures indicated a certain number, that number would be used, even if the article text did not state how many chimpanzees had been used.

\section{Citations to examples}

Citations to relevant articles are provided where the results and conclusions from the survey are discussed. Because of the large survey base, however, it was impractical to cite all the relevant articles for each given statement. Therefore, where multiple articles from the survey could be cited as examples, this article provides, by way of examples, citations to two articles, in order to constrain the page and citation numbers of this survey. More than two examples may be given, if the examples provide a representative range of studies.

\subsection{Review of approved mAb drugs}

The review of the regulatory approvals of $\mathrm{mAb}$ drugs in the United States was based on documents made available on the FDA web site. The web site provides, however, only selected parts of the agency record, as opposed to the entire record, and even the available documents have redactions made pursuant to the exemptions in the Freedom of Information Act. A number of Freedom of Information Act requests have been made to the FDA for additional information regarding a number of these 
drug approvals, but by press time no answer had yet been received from the agency. Therefore, this analysis is by necessity restricted to the information currently available on the FDA web site. One might presume, however, that the selected records the FDA makes available are those that it considers to contain the most relevant information regarding the approval.

Both in the United States and internationally, the accepted nomenclature classification for nonproprietary drugs designates the suffix "-mab" for monoclonal antibody drugs (World Health Organization, 2009). Therefore, our review and analysis of FDA approvals was restricted to drugs specifically identified as mAbs through that suffix. Other drugs also have been approved that have many functional similarities with mAbs. These drugs combine the target-specificity of a mAb as a drug-delivery vehicle, with a small molecule or a fusion protein to target another molecule, such as a cytokine. Such other classes of drugs fall outside this review.

\section{Results}

\subsection{Survey of scientific articles}

\subsubsection{Frequency of articles}

The 193-article base was first sorted by publication year, and the articles then were grouped by 5 -year periods into six groups to show what trends, if any, could be observed in this regard. Figure 1 shows as the first data series the number of all articles in a given group. As the second data series, Figure 1 shows the numbers of articles that provided specific information regarding the number of chimpanzees used in the research in question. This issue will be relevant, as we will discuss later the numbers of chimpanzees used in mAb research.

In the first half of the 1980's, the number of published articles was only 19, as mAb technology was still new and not within the reach of all researchers. The number grew rapidly to 58 in the latter part of that decade, but then started a decline. In the latter part of the 1990's, that decline was nearly $50 \%$ of the early 1990's (28 v. 52), and reached even lower values, 19 and 21, for the most recent decade.

The proportion of peer-reviewed "chimpanzee mAb" articles of all peer-reviewed $\mathrm{mAb}$ articles also continues to decrease. The percentage of chimpanzee mAb articles of a PubMed search for "monoclonal AND antibody" (accessed 01/31/11 and excluding reviews) for the period of $1981-1990$ is $0.14 \%$, and for the period of $1991-2000,0.1 \%$. For 2001-2010, that percentage is less than $0.07 \%$.

\subsubsection{Number of chimpanzees involved in the studies}

Aggregate numbers

To arrive at aggregate numbers, only those articles where a specific number was given for the chimpanzee(s) were included in the following analysis. Figure 2 shows two data series in this respect. The first gives the aggregate number of chimpanzees in a given period, based on a straightforward summing up of the relevant numbers. During 1986-1995, however, there were five articles that each discussed studies where blood samples were obtained from a very large number of chimpanzees, and it seemed possible that these five articles might skew the figure. Therefore, the second data series presents the aggregate chimpanzee numbers without these extreme cases.

For the first, unadjusted data series Figure 2 shows a rapid climb from the early 1980's over the next decade to reach the peak of 1,213 chimpanzees in the period of 1991-1995. The next 5-year period saw a sharp drop to 91 chimpanzees, and the most recent decade shows that 28 and 33 chimpanzees, respectively, were used in the studies published in its 5-year periods.

On the other hand, if we were to look for the number of individual chimpanzees used in these studies, it is likely that some

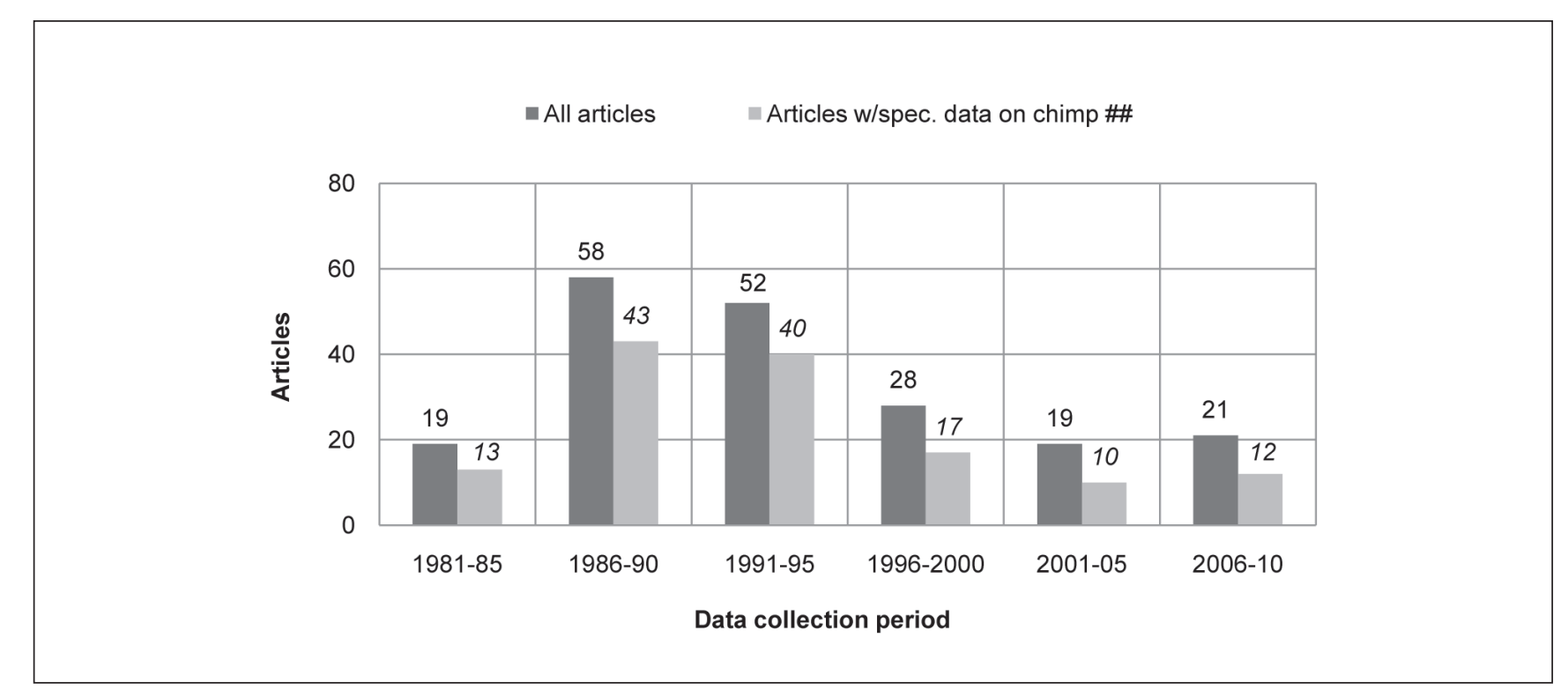

Fig. 1: Frequency of articles dealing with live chimpanzees in mAb research 
All articles w/ specific data $\square$ Same, but w/o studies using 100+ chimpanzees

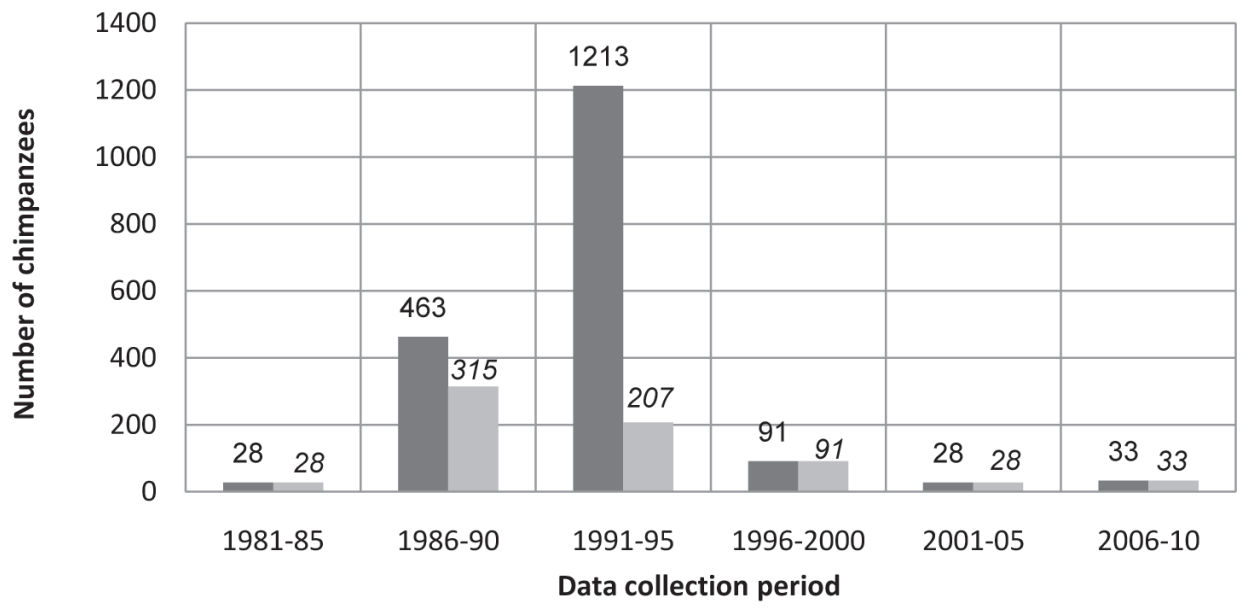

Fig. 2: Number of chimpanzees in mAb studies

chimpanzees had been used in multiple studies. From that perspective, the total aggregate number may be an over-count of the individuals. Unfortunately, the often sketchy information provided in the surveyed articles does not permit a reliable estimate of the individual chimpanzee number based on all of the published studies.

\section{Number of chimpanzees in a given experiment}

The lack of specific information regarding the chimpanzees used in the studies makes it impractical to attempt an acrossthe-board estimate of the numbers of chimpanzees in individual studies. Based on those articles that gave a specific number, however, the following counts could be made (Tab. 1).

The numbers in the table suggest several observations: For example, experiments in the most recent decade (2001-10) generally used smaller numbers of chimpanzees than in the previ-

Tab. 1: Number of chimpanzees used in an individual study* *Where the article describing the study gives a specific number.

\begin{tabular}{|c|c|c|c|c|c|c|}
\hline Period \# & $\mathbf{1 - 2}$ & $\mathbf{3 - 5}$ & $\mathbf{6 - 1 0}$ & $\mathbf{1 1 - 2 0}$ & $\mathbf{2 1 - 9 9}$ & $\mathbf{> 1 0 0}$ \\
\hline $\mathbf{1 9 8 1 - 1 9 8 5}$ & 4 & 4 & 3 & 1 & 1 & 0 \\
\hline $\mathbf{1 9 8 6 - 1 9 9 0}$ & 19 & 12 & 5 & 3 & 3 & 1 \\
\hline $\mathbf{1 9 9 1 - 1 9 9 5}$ & 16 & 7 & 7 & 4 & 2 & 4 \\
\hline $\mathbf{1 9 9 6 - 2 0 0 0}$ & 6 & 6 & 2 & 3 & 0 & 0 \\
\hline $\mathbf{2 0 0 1 - 2 0 0 5}$ & 4 & 3 & 3 & 0 & 0 & 0 \\
\hline $\mathbf{2 0 0 6 - 2 0 1 0}$ & 8 & 3 & 1 & 0 & 0 & 0 \\
\hline
\end{tabular}

ous decades. Also, in the last decade, no studies used more than 10 chimpanzees. The most common number across the three decades (in $42 \%$ of the articles) is one or two chimpanzees per study. The peak numbers of chimpanzees used per study occurred in 1991-95.

\subsubsection{Topics of research}

The 1980's witnessed the application of the new mAb technology to biomedical sciences. This was reflected in survey articles, in that many of the reviewed $\mathrm{mAb}$ articles dealt with basic-type mAb science, such as comparing antigenic properties of red and white blood cells (Neubauer et al., 1981; Socha and Ruffie, 1990). While many studies found cross-reactivity with human and chimpanzee epitopes, such findings were by no means uniform, and a number of studies confirmed differences between humans and chimpanzees (Hammond and Robinson, 1984; Palmer et al., 1987; Lu et al., 1987). In some cases, a mAb would not bind to chimpanzee antigens, although it did so with human and other non-human primate antigens (Dowell et al., 1984; Shaw, 1986).

In the latter part of the 1980's, the mAb technology was being applied to increasingly diverse components of the immune system and to infectious disease investigations, such as AIDS, hepatitis, and leukemia (Iwarson et al., 1985; Winton et al., 1985; Goudsmit et al., 1987). It was thought that if conventional vaccines could successfully prevent disease by building humoral immune memory, mAbs could be used in a similar manner to prevent newly-identified viral diseases. The chimpanzee was believed to be a useful animal model to researchers because many mAbs would bind both to human and chimpanzee antigens, and laboratory reagents developed for human use thus could be used in chimpanzee studies (Ehrlich et al., 1988; Moller et al., 1990). For example, the chimpanzee was used extensively to search for immunomodulatory mAbs and to 
observe infusion reactions following their administration (Van Meurs and Jonker, 1986; Jonker et al., 1988).

In the 1990 's, many published articles on mAb studies with chimpanzees continued to focus on properties of the serum and the immune response. Again, while similarities were found between the human and the chimpanzee in these respects, many articles also noted differences (Blancher and Socha, 1991; Socha and Moor-Jankowski, 1993). Some researchers specifically focused on erythrocytes and related molecules, and their studies typically compared characteristics of samples from humans, great apes (hominoids), and other non-human primates (Blancher et al., 1992; Socha et al., 1993). Nine articles described the use of chimpanzees in endotoxemia experiments, attempting to explain molecular causes of sepsis (Levi et al., 1994, 1998). A number of articles investigated immunomodulatory properties of lymphocytes and cytokines, including reactions that frequently result from the infusion of therapeutic mAbs (Rao et al., 1991; Logdberg et al., 1994). Articles from this decade also frequently deal with research on HIV- and hepatitis-related mAbs (Emini et al., 1992; Sawada et al., 1995; Zhou et al., 1999).

In the 2001-2010 decade, perhaps partly due to the smaller number of articles, the distribution of study topics was more heterogeneous. While the procedural features of the studies varied, the majority of articles could be broadly categorized as dealing with infectious disease studies (Esumi et al., 2002; Men et al., 2004; Hatziioannou et al., 2009). A few articles discuss the effectiveness or properties of immunomodulatory mAbs (Newman et al., 2001; Boon et al., 2002).

\subsubsection{Experimental procedures}

\section{Chimpanzee experience in general}

Typically, the information in the articles regarding the treatment of chimpanzees was minimal or non-existent. The majority did not describe the type or manner of procedures applied to the chimpanzees, their health status before, during, or after the experiment, or conditions in which they were housed and cared for. Usually, the information only dealt with the type of biological sample(s) obtained from the chimpanzee. Only a small minority of the articles provided information regarding the administration, type and length of anesthesia, measurements of clinical data, and apparent health and well-being of the chimpanzees before, during, and immediately after the experiment.

\section{Blood samples}

All of the experiments appeared to involve blood draws either before, during, and/or after the experiment, even if this was not explicitly stated in the article. These were required either for the analysis of the mAbs and their effects, or for observing the infection stage of the chimpanzees, and in general to determine the health status of the chimpanzees. Even if a blood draw was explicitly mentioned, in most cases it was a short statement without further elaboration, such as: "Serum samples from two chimpanzees ... were tested ..." (Maillard et al., 2001), or "[a] blood sample was collected weekly from each animal ..." (Men et al., 2004).

Sometimes, a blood sample appeared to be the only invasive procedure, such as in many studies in the 1980's where the analysis of the components of serum or plasma was the main objective of the studies. In many cases, however, the study required a series of blood draws, either within a relatively short time period (such as 2-12 weeks) but also for extended periods, as in many hepatitis experiments. In those studies, weekly or biweekly draws would be done for over a year or several years (Zhang et al., 2006; Ogata et al., 1993).

\section{Tissue samples and biopsies}

In addition to blood draws, many studies also required more invasive procedures, such as one or more bone marrow aspirations, or biopsies of lymph nodes and/or liver, and/or other tissue samples (such as kidney, spleen, or lung), and catheterization for urine samples (Kim et al., 2008; Geijtenbeek et al., 2001; Borke et al., 1987; Thirkell et al., 1990). The type of biopsy (such as needle, laparoscopic, or surgical biopsy) was usually not specified. In the case of long-running studies (Goncalvez et al., 2008), a chimpanzee might be subjected to dozens of liver biopsies or other tissue biopsies (Bukh et al., 2008).

Some more recent studies involved bone marrow sampling (single or repeated), by bone marrow aspiration or biopsy (Schofield et al., 2000; Men et al., 2004; Goncalvez et al., 2008). Bone marrow is the source of blood cells (hematopoiesis) and, due to advances in biochemical techniques, may provide ever-increasing amounts of information about the physical and chemical characteristics of blood cells and related molecules. While a less frequent occurrence, researchers in a 2002 reproductive study obtained samples of the chimpanzee spleen, external and internal reproductive tract, and sperm from a contractor housing primates (McCauley et al., 2002). In a 1982 study, spleens were removed entirely from 19 chimpanzees before they were infected with malaria in order to observe the immune response in the absence of this important lymphoid organ (Nardin et al., 1982).

\section{Infectious agents}

A large portion of the studies involved infecting chimpanzees with a bacterial or viral pathogen that would cause an infectious disease, or injecting them with a toxic compound that would cause typical symptoms of the disease in question. HIV and hepatitis were by far the most frequent pathogens used in this manner (Wang et al., 1999; Bukh et al., 2008). Others included smallpox, Dengue fever, anthrax, human T-cell lymphoma virus (HTLV), herpes, respiratory syncytial virus, tuberculosis, encephalitis, and malaria (Chen et al., 2006a,b; Men et al., 2004; Arp et al., 1996; Blewett et al., 1999; Crowe et al., 1994; Franzoso et al., 1993; Goncalvez et al., 2008; Klotz et al., 1996). In a series of experiments, $E$. coli was used in amounts sufficient to trigger symptoms of endotoxemia that would mimic early phases of sepsis (Levi et al., 1998; Jansen et al., 1995). In other experiments chimpanzees would be induced to develop plaques in brain tissue, asserted to be similar to those found in Creutzfeld-Jacob disease (Toh et al., 1985).

Generally, amounts of infectious agents used in the studies were significantly larger than what had previously been found to be sufficient to cause infection in chimpanzees. (The infectivity is measured in CID - a chimpanzee infectious dose suf- 
ficient to infect at least $50 \%$ of the chimpanzees that the doses had been tested on.) For example, one recent study infected the chimpanzees with 1000 CID-50 of hepatitis B virus (HBV), and 55 weeks later, one chimpanzee was challenged again with the same dose of HBV (1000 CID-50) (Zhang et al., 2006). The studies do not discuss reasons for a particular dose.

The methods used to introduce the infectious micro-organism into chimpanzees varied. Frequently, chimpanzees were injected intravenously or intramuscularly with the virus or bacteria, but some studies described the method of infection as "intrahepatic" or "intratracheal" (Emini et al., 1992; Franzoso et al., 1993; Forns et al., 2000; Meunier et al., 2008).

In several studies, chimpanzees were infected with multiple types of a disease, so-called "superinfection." For example, in some experiments involving hepatitis or Dengue fever vaccines, the chimpanzees were infected with all known genotypes of the virus in order to induce the chimpanzees to produce a wider repertoire of virus-specific antibodies (Men et al., 2004; Schofield et al., 2002).

Vaccines and $m A$ b infusions

Passive immunization confers protection from pathogens or toxins through antibodies that neutralize those pathogens. Many chimpanzee experiments, therefore, investigated the use of mAbs as a vaccine or as a means to neutralize infectious or toxic agents. This requires that large amounts of mAbs be introduced into a chimpanzee, which would typically be accomplished by infusion (Ogata et al., 1993; Wang et al., 1999). Similarly, large amounts of mAbs are needed to block molecular receptors sufficiently, as in the case of mAbs that were developed to repress immune function for therapeutic reasons (such as in leukemia or autoimmune diseases) (Fishwild et al., 1999; Rao et al., 1991).

It was recognized that $\mathrm{mAb}$ and infusion procedures could cause unpleasant side effects, and sometimes even life-threatening complications. Typically, during an infusion a drug or another chemical compound is administered intravenously through a needle or a catheter while the animal is under some type of anesthesia, such as ketamine and/or gaseous anesthesia $\left(\mathrm{N}_{2} \mathrm{O}, \mathrm{O}_{2}\right.$, halothane mixture) (Jonker et al., 1993). In the 1990's, a number of chimpanzee studies investigated different mAb variants and different doses that might minimize or eliminate such undesirable effects. In these studies, some chimpanzees demonstrated severe adverse effects, such as in a study where a chimpanzee developed a fever of $40^{\circ} \mathrm{C}\left(104^{\circ} \mathrm{F}\right)$, requiring treatment with ice packs (Rao et al., 1991). Some mAbs brought on other severe symptoms, such as shock-like symptoms, respiratory difficulties, irregular heartbeat, cyanosis, jerking, and/or vomiting (Parleviet et al., 1990; Jonker et al., 1993; Harpprecht et al., 1990). If, on the other hand, side effects were deemed minimal or transitory, the article would merely note that fact without further elaboration. One endotoxemia study noted that while there were changes in some cell populations obtained from the chimpanzees, these changes were consistent with a "stress response to the infusion procedure" (Fishwild et al., 1999).

Experiments to develop vaccines for active immunity would typically require multiple injections, such as an initial immuniza- tion and subsequent booster(s) (Chen et al., 2006b). In a hepatitis C study, a chimpanzee was vaccinated 13 different times with epitope(s) and adjuvants over a 34-week period, and was challenged twice with HCV (Esumi et al., 2002). In some cases, such as those involving DNA vaccines, a series of immunizations would be performed on each chimpanzee (Forns et al., 2000).

\subsubsection{Chimpanzee health and welfare}

\section{Pre-study health status}

The reviewed articles typically contained little or no information pertaining to the chimpanzees' past or overall health and welfare that might have influenced the mAb study results. Only a few articles gave information on age, sex, weight, and possible prior exposure to infectious disease(s) (Crowe et al., 1994; ten Cate et al., 1993). Since the early 1990 's, many articles - approximately $25 \%$ - contained a statement that the procedures observed in the study complied with the applicable institutional animal care guidelines (Chen et al., 2006a; McCauley et al., 2002).

\section{Anesthesia and analgesia}

As a rule, there was no mention of any anesthesia in the context of bleeds. In some instances where the procedure appeared to be more complex, such as a lengthy process that combined bleeds, infusions and/or biopsies, ketamine chloride or nitrous oxide and halothane were reported as having been administered (Geijtenbeek et al., 2001; van der Poll et al., 1996; Levi et al., 1998). It is possible, of course, that anesthesia and analgesia were provided in other studies, but the description of the procedure lacked such information.

\section{Control animals}

The experience of chimpanzees used as control animals in infectious disease studies also merits consideration. Like experimental chimpanzees, they were subject to frequent blood draws, biopsies and other experimental procedures. However, while chimpanzees in experimental groups frequently suffered from side effects of pathogenic infection or toxemia, in some cases $\mathrm{mAb}$ therapy might at least temporarily ameliorate the symptoms of the disease. Control chimpanzees did not have this benefit (Forns et al., 2000; Sawada et al., 1995).

\section{Origin of chimpanzees}

The majority of chimpanzees currently in U.S. laboratories were captured from the wild or are descendants of wild-caught chimpanzees. Some chimpanzees are former pets or were used in the entertainment industry prior to being given up to research. Importation of wild-caught chimpanzees into the US has been prohibited since 1977. While most U.S. and European researchers do not use chimpanzees in situ in Africa for their mAb studies, our review indicated that some studies describe such research. For example, one study obtained blood samples from Central and West African chimpanzees in order to compare hepatitis B virus strains, and after identifying an unusual strain, the researchers concluded that more testing in central Africa needed to be done to confirm the results of this study (Takahashi et al., 2001). Other studies used chimpanzees and other hominoids housed at an institute in Franceville, Gabon (Apoil et al., 1997; Tournamille et al., 2004). 


\subsection{Approval of mAb drugs}

\subsubsection{Requirements for non-clinical animal studies}

The International Conference on Harmonization of Technical Requirements for Registration of Pharmaceuticals for Human Use (ICH), to which the United States is a party, has issued guidance regarding the preclinical (non-clinical) safety evaluation of biotechnology-derived drugs (biologics) (ICH, 1997). Section 3.3. of this guidance (S6) states specifically that the relevant animal species for testing of monoclonal antibodies should express the desired epitope and demonstrate a similar tissue crossreactivity profile as for human tissues. Such testing is intended to optimize the ability to evaluate toxicity arising from the binding to the epitope and any unintentional tissue cross-reactivity. The ICH guidance recognizes, however, that in the absence of a relevant animal model, relevant transgenic animals that express the human receptor or the use of homologous proteins should be considered (ICH, 1997).

Consistent with its statute and the ICH guidance, the FDA has not set forth any hard-and-fast requirements regarding the animal species to be used in preclinical testing. Specifically, there is no FDA requirement that would obligate pharmaceutical companies to use the chimpanzee. A flexible regulatory approach is necessary because each mAb product is unique, and therefore the most appropriate scientific means for testing safety and toxicity must be arrived at through individual analysis in each case (Bhogal et al., 2008).

\subsection{2 mAb drug approvals}

The documents publicly available on the FDA web site show that the agency approved for distribution the first mAb drug, muromonab OKT3, in 1992 (FDA, 2010a) ${ }^{1}$. By 12/31/10, the FDA had approved $32 \mathrm{mAb}$ drugs, of which 28 currently remain on the market (FDA, 2010b).

Of the 32 approvals, the FDA web site has information about preclinical animal studies for 28 drugs. No information on animal studies could be found for muromonab-OKT3, fanolesomab (discontinued in 2008), nofetumomab, and trastuzumab. Table 2 lists these 28 drugs chronologically based on the approval date. The table also indicates which species, according to the available information, were used in preclinical studies.

The information made available by the FDA indicates that the preclinical information for only three of the approved drugs involved chimpanzees. Of those three drugs, only in the case of infliximab was a more detailed description available of the type of studies the chimpanzee was used for - and the drug still remains on the market. For the majority of mAbs on the market, cynomolgus macaques, in addition to mice, have been used as the preferred species to test toxicology.

The FDA data indicate that of the 28 drugs, only three have been withdrawn from the market, and for one of these, daclizumab, the withdrawal was due to business reasons rather than serious adverse safety issues. The only two withdrawals that involved significant safety issues for human patients involved the drugs efalizumab and gemtuzumab, each of which had used the chimpanzee in its preclinical studies.

\footnotetext{
1 Other available information indicates that the very first FDA approval for this drug dates from 1986, but it is not clear whether this statement refers to the approval for investigation (IND) or distribution (Strohl, 2009).
}

Tab. 2: Therapeutic mAb drugs approved by the FDA with preclinical animal test information available

\begin{tabular}{|c|c|c|c|}
\hline Drug / Trade name & Year approved / discontinued & Biological target & Species used in preclinical studies \\
\hline Abciximab / ReoPro & 1994 & Platelet glycoprotein & Mouse, rat, dog, monkey, baboon \\
\hline Capromab / ProstaCint & 1996 & $\begin{array}{l}\text { A glycoprotein expressed } \\
\text { by the prostate specific } \\
\text { membrane antigen }\end{array}$ & $\begin{array}{l}\text { Mouse, cynomolgus monkey, } \\
\text { rat, rabbit }\end{array}$ \\
\hline Imciromab / Myoscint & 1996 & Myosin bound to DTPA & Rat, dog, non-human primate \\
\hline Daclizumab / Zenapax & 1997 / 2009 (not a safety issue) & IL-2R or CD25 & Cynomolgus monkey \\
\hline Rituximab / Rituxan & 1997 & CD20 & Rat, cynomolgus monkey \\
\hline Basiliximab / Simulect & 1998 & IL-2R (CD25) & Rhesus, cynomolgus monkey \\
\hline Infliximab / Remicade & 1998 & TNF & $\begin{array}{l}\text { Mouse, rat, beagle dog, chimpanzee, } \\
\text { cynomolgus monkey }\end{array}$ \\
\hline Palivizumab / Synagis & 1998 & RSV (F protein, site A) & $\begin{array}{l}\text { Cotton rat, cynomolgus monkey, } \\
\text { rabbit }\end{array}$ \\
\hline Gemtuzumab / Mylotarg & $2000 / 2010$ & CD33 & $\begin{array}{l}\text { Mouse, rat, cynomolgus monkey, } \\
\text { chimpanzee, beagle dog }\end{array}$ \\
\hline Alemtuzumab / Campath & 2001 & CD52 & Mouse, cynomolgus monkey \\
\hline
\end{tabular}




\begin{tabular}{|c|c|c|c|}
\hline Drug / Trade name & Year approved / discontinued & Biological target & Species used in preclinical studies \\
\hline Adalimumab / Humira & 2002 & TNF & $\begin{array}{l}\text { Mouse, rat, guinea pig, rabbit, dog, } \\
\text { cynomolgus monkey }\end{array}$ \\
\hline $\begin{array}{l}\text { Ibritumomab tiuxetan / } \\
\text { Zevalin }\end{array}$ & 2002 & CD20 & Mouse, rat, cynomolgus monkey \\
\hline Efalizumab / Raptiva & $2003 / 2009$ & CD11a & Mouse, chimpanzee \\
\hline Omalizumab / Xolair & 2003 & $\lg E$ & Cynomolgus monkey \\
\hline Tositumomab / Bexxar & 2003 & CD20 & Cynomolgus monkey \\
\hline Bevacizumab / Avastin & 2004 & VEGF & $\begin{array}{l}\text { Mouse, rat, rabbit, } \\
\text { cynomolgus monkey }\end{array}$ \\
\hline Cetuximab / Erbitux & 2004 & EGFR & $\begin{array}{l}\text { Mouse, rat, rabbit, } \\
\text { cynomolgus monkey }\end{array}$ \\
\hline Natalizumab / Tysabri & 2004 & $a 4 \beta 1$ and $\alpha 4 \beta 7$ integrins & $\begin{array}{l}\text { Mouse, guinea pig, } \\
\text { cynomolgus monkey, rhesus }\end{array}$ \\
\hline Panitumumab / Vectibix & 2006 & EGFR & $\begin{array}{l}\text { Mouse, rat, rabbit, } \\
\text { cynomolgus monkey }\end{array}$ \\
\hline Ranibizumab / Lucentis & 2006 & VEGF & $\begin{array}{l}\text { Rabbit, cynomolgus monkey, } \\
\text { guinea pig }\end{array}$ \\
\hline Eculizumab / Soliris & 2007 & CD52 & Mouse \\
\hline Certolizumab pegol / Cimzia & 2008 & TNF & Mouse, rat, cynomolgus monkey \\
\hline Canakinumab / llaris & 2009 & $\mathrm{IL}-1 \beta$ & Mouse, marmoset \\
\hline Golimumab / Simponi & 2009 & TNF & Mouse, cynomolgus monkey, rhesus \\
\hline Ofatumubab / Arzerra & 2009 & CD20 & Mouse, cynomolgus monkey \\
\hline Ustekinumab / Stelara & 2009 & IL-12, IL-23 & Mouse, cynomolgus monkey \\
\hline Tocilizumab / Actemra & 2010 & IL-6R & Mouse, cynomolgus monkey \\
\hline Denosumab / Prolia & 2010 & RANKL & Mouse, rat, cynomolgus monkey \\
\hline
\end{tabular}

\subsubsection{Infliximab}

Infliximab (trade name: Remicade ${ }^{\mathrm{TM}}$ ) is a chimeric mouse/human hybrid mAb. It was initially approved in 1998 to control Crohn's disease, a chronic inflammatory bowel disease, and in 2004, it was additionally approved to treat rheumatoid arthritis. In both diseases, infliximab reduces chronic inflammatory symptoms by preventing the binding of tumor necrosis factor alpha $(\mathrm{TNF} \alpha)$ to its receptor and thus inhibits the activity of TNF $\alpha$ (FDA, 1998).

Preclinical studies were conducted on mice, chimpanzees, cynomolgus monkeys, rats, rabbits, and beagle dogs. In addition, cross-reactivity studies included the baboon, macaque, tamarin, and pig. The chimpanzee TNF $\alpha$ was the only one, besides human, that bound infliximab, and therefore, in addition to the pharmacokinetic studies, the chimpanzee was used in limited safety studies. These involved 3-5 consecutive doses, each series with a different dosing strength. The FDA review notes that the chimpanzee safety studies conducted in 1993 showed no signs of toxicity. The documents also state, however, that because the applicable restrictions prohibit necropsies on chimpanzees, the studies could not provide histopathological data and were limited to clinical chemistry, hematology assessments, and clinical observations (FDA, 1998).

Transgenic mice and surrogate antibodies were the main means of providing preclinical safety and efficacy data. The immunogenicity testing for infliximab was performed using the cynomolgus monkey and the mouse. Efficacy was not tested in the chimpanzee, although some immune response in them was noted.

In contrast to preclinical studies, the clinical trials disclosed a number of severe and less severe adverse effects in patients that were not observed in the chimpanzee model. General side effects included infusion reactions and immunogenicity, and hypersensitivity reactions that bring on headache, nausea, and arthralgia (FDA, 1999). Other adverse effects included immunosuppression (possibly predisposing to tuberculosis), reduced 
blood cell and platelet counts, malignancies, and elevated liver enzymes. In August 2009, the FDA issued a safety alert to include leukemia and psoriasis among possible risks associated with the use of a number of TNF blockers, including infliximab (FDA, 2009b).

\subsubsection{Efalizumab}

Efalizumab (trade name: Raptiva ${ }^{\mathrm{TM}}$ ) is an immunosuppressant that the FDA approved in 2003 for treating psoriasis. Although the FDA approval letter mentions that the preclinical studies included chimpanzees, the available documents provide only fragmentary additional information regarding these studies.

The chimpanzee apparently was used in cross-reactivity studies, since the documents state that efalizumab binds only the human and chimpanzee immune molecule (LFA-1) that is the target (Genentech, 2003; Bauer et al., 1999). The chimpanzee also was used in some pharmacokinetics and toxicology studies. The FDA documents note that chimpanzees were exposed to a 6-month study involving up to a 339 -fold dose of the mAb, compared to the projected human dose, to evaluate its toxicology (Genentech, 2003). They also note that the immune response of chimpanzees treated with this mAb was reduced after injection of tetanus toxin (FDA, 2003). Due to the lack of direct cross-reactivity of efalizumab in mice, a surrogate murine equivalent was developed to test its efficacy (Genentech, 2003).

When efalizumab was approved, the FDA required Genentech to conduct seven additional clinical studies. However, reports of serious side effects caused Genentech, following urging by the FDA, to withdraw the drug from the market in 2009 before all of these trials had been completed and fully analyzed. A similar measure took place in the European Union. The FDA notice regarding the withdrawal mentions specifically three incidents of progressive multifocal leukoencephalopathy, that is, inflammation of brain matter due to viral infection and/or immune deficiency (FDA, 2009c). Other adverse effects noted in clinical trials and post-marketing practice included anemia and thrombocytopenia (deficiency of blood platelets, resulting in bleeding); inflammatory immune-mediated events; serious infections; and worsening of psoriasis (FDA, 2003).

\subsubsection{Gemtuzumab ozogamicin}

Gemtuzumab ozogamicin (trade name: Mylotarg ${ }^{\mathrm{TM}}$ ) is a chemotherapeutic drug for treating a form of bone marrow cancer (acute myeloid leukemia, or AML). It consists of two parts, a monoclonal antibody and a cytotoxin. The monoclonal antibody is specific for the CD33 molecule that is expressed on the surface of early myeloid leukemia cells and leukemic cells of a majority of AML patients. The antibody is combined with a cytotoxic antibiotic, calicheamicin, which the antibody delivers to the cancer cells in order to destroy them.

The preclinical toxicity review involved mice, rats, and monkeys whose liver toxicity levels appeared to be concordant with humans (FDA, 2000). The FDA documents indicate, however, that early toxicity studies also involved chimpanzees and dogs. The sole chimpanzee study involved a 2-hour infusion of the drug into two chimpanzees who then were monitored for two weeks. Although some abnormalities were noted, it was assumed these may have been associated with the ketamine anesthesia (FDA, 1996).

The FDA granted gemtuzumab accelerated approval in May 2000 , but the sponsoring company was required to conduct additional clinical trials after the approval to confirm the drug's benefit. One of these post-approval clinical trials raised new concerns about the drug's safety, as it was associated with a serious liver condition (veno-occlusive disease), which can be fatal. Furthermore, the company failed to demonstrate that the drug was of sufficient clinical benefit to patients enrolled in trials. Therefore, on June 21, 2010, the FDA informed healthcare professionals and the public that the company was withdrawing gemtuzumab from the market (FDA, 2010c).

\section{Discussion}

\subsection{Outlook on the future use of the chimpanzee}

What conclusions might this review suggest regarding the use of the chimpanzee in biomedical research? For example, what circumstances might have led to the apparent sharp reduction in the use of chimpanzees? While some use likely continues in private laboratories that do not publish their experiments, a number of reasons point to a real and substantial reduction. First, advances in biomedical technology and tools offer researchers alternative means of carrying out sophisticated investigations at a molecular level through in vitro and in silico studies. Second, steep financial costs associated with the chimpanzee model are also a pragmatic and powerful factor. Of a number of possible topics, we will look here briefly at the genetic and biological suitability of the chimpanzee, alternative options for preclinical studies, and economic considerations in drug development planning.

While ethical considerations are beyond the scope of this article, they tangibly influence whether chimpanzees are used and if so, the ways in which chimpanzee research currently is conducted. Public opinion, which increasingly supports animal welfare and non-animal research options, expresses societal views in this respect (National Science Board, 2002; The Pew Research Center for the People \& the Press and The American Association for the Advancement of Science, 2009). Both the endangered status of the chimpanzee in the wild and ethical issues appear to influence the research policies of institutions in many countries that restrict the use of the chimpanzee. Finally, socially responsible companies find it possible to be responsive both to societal concerns and economic concerns of their shareholders by using research methods that do not involve the chimpanzee.

\subsection{Suitability of the chimpanzee model}

Historically, the chimpanzee model has relied on the phylogenetic closeness of chimpanzees to humans - first on the morphological and phenotypic level and subsequently on the research of blood components, including antigen epitopes (Fridman, 2002). Indeed, in the 1980's and 1990's, much of $\mathrm{mAb}$ research that used the chimpanzee was based on these as- 
sumptions and their study by immunological and cellular techniques. Today, however, molecular biology and the technology it employs have moved on to other, more effective and successful research techniques and strategies, as has the scientific understanding of many diseases.

The oft-repeated " $1 \%$ difference" in our respective genomes may make it appear that the difference between humans and chimpanzees is negligible, but even these differences are actually significant both on the molecular and the gene expression level. For example, large-scale rearrangements, including deletion of entire genes, increase the DNA differential by several percentage points (Perry et al., 2008). Of the specific gene families, transcription factors that regulate gene expression and immune response genes have undergone significant development since chimpanzee and human genomes diverged (Chimpanzee Sequencing and Analysis Consortium, 2005). In addition, the characterization of differences resulting from our respective epigenetic and non-coding regulatory DNA has barely begun (Weinstock, 2007).

Key differences in human and chimpanzee immune functions continue to be documented. Human lymphocytes appear to be over-reactive compared to those of the chimpanzee, which is manifested in the outcome of some chronic diseases, such as HIV and hepatitis. While it is not entirely clear what the biological basis for this difference is, it has recently been shown that this over-reactivity correlates with decreased levels of inhibitory sialic acid-recognizing Ig-superfamily lectins on human T and B cells (Soto et al., 2010). Among other carbohydrates, these molecules have important functions in protein conformation and solubility and effector immune responses (Nguyen, 2006). Also, NK cells that serve critical functions in immunity are significantly different in humans and chimpanzees (Abi-Rached et al., 2010).

While the PK \& PD data of small molecule drug clearance and metabolism in the chimpanzee may in many respects be comparable to that in humans, there are also many differences, such as in the P450 enzyme activity and extrahepatic clearance, so that a simple assumption of overall comparability would be difficult (Wong et al., 2004). Moreover, the PK \& PD of a mAb drug may vary by many additional factors, including antigen expression and distribution and downstream immune responses, as the $\mathrm{mAb}$ in question may have pleiotropic effects (Gibson et al., 2009).

The history of approved mAb drugs that used the chimpanzee in their preclinical studies points to the difficulty of predicting results in human patients based on this model. It would not be appropriate to suggest that the preclinical chimpanzee studies should have disclosed all of the serious adverse effects found later in humans. Because we deal here with only three drugs, any statistical conclusions are not possible, either. Nevertheless, it may not be prudent to dismiss without further investigation, as a mere coincidence, the fact that two out of these three drugs were withdrawn from the market because of their serious adverse effects on human patients, particularly as this has not happened with the other approved mAb drugs.

\subsection{Alternative models}

Reactivity of the mAb to the target antigen is a key concern when an animal model is selected for testing of a mAb drug. In addition to the genetic sequence of the epitope itself, conformational and other structural issues affecting the mAb are important. Preliminary in vitro testing usually is performed to determine the likely in vivo function of the $\mathrm{mAb}$ in various species. For many mAbs, orthologous epitopes can be found among a number of species, and only rarely would the chimpanzee appear to be the only species with cross-reactivity to the human $\mathrm{mAb}$ drug candidate. Indeed, a mAb may be specific only to humans. Such was the case with eculizumab, and in preclinical studies a murine surrogate was used to test the drug's toxicological effects (FDA, 2007).

To achieve the desired therapeutic effect, there may be two or more alternative mechanisms that a mAb drug may employ. For example, there are currently four approved mAb drugs to treat TNF $\alpha$-related conditions. The first drug, infliximab, was approved in 1998 and used the chimpanzee in the preclinical phase. Three other such drugs (adalimumab, certolizumab and golimumab) subsequently have been approved without any indication in the FDA documents of the use of the chimpanzee. Similarly, efalizumab was approved to treat psoriasis, using the chimpanzee in its preclinical studies. In contrast, ustekimumab, another psoriasis drug approved a few years later, did not rely on the chimpanzee model (FDA, 2009a).

Even if the chimpanzee were the only non-human species that recognizes a human $\mathrm{mAb}$, satisfactory preclinical testing may be possible through alternative means. Transgenic rodents may in some cases express the relevant human antigen in a manner that allows PK \& PD and toxicity studies. A surrogate mAb also may cross-react and function in another animal species in a manner similar to the mAb drug. Both of these approaches were used in the preclinical studies for infliximab, which only crossreacted with the human and the chimpanzee. For infliximab, a transgenic mouse was produced expressing human TNF $\alpha$ that could then bind the mAb antagonist. Also, an anti-mouse TNF $\alpha$ antibody surrogate was used in neutralization studies to study the efficacy of the proposed mAb (FDA, 1998).

Increasingly sophisticated in vitro techniques are used to perform tests on relevant human cell lines. In many respects, these tests are more useful than animal preclinical tests that may leave open the questions of whether the markers used in them are the most relevant ones for the purpose, and whether the results are ultimately translatable to the human patient. Both pharmaceutical firms and regulatory authorities recognize the benefits of research techniques that provide direct information about the function of the drug candidate in the human patient.

Before recombinatorial genetic engineering and later $\mathrm{mAb}$ technology advances, chimpanzee antibodies sometimes were regarded as a surrogate in search for mAbs that could be used in humans. For example, chimpanzees could be infected with a pathogen in order to induce them to produce neutralizing antibodies that, in turn, could then be analyzed for optimal use in a human vaccine (Crowe et al., 1994). Today, this use of the chimpanzee is made redundant by a number of other, more ef- 
ficient means of developing mAbs with desired characteristics (Almagro and Strohl, 2009).

In sum, a forward-looking mAb development plan may be able to ensure that the use of the chimpanzee is unnecessary. Current genetic information of candidate mAbs and their respective antigens in various species may permit the selection of a mAb and an animal model, other than the chimpanzee, with appropriate cross-reactivity and expression of antigens. In addition, in vitro testing may provide critical information about the function of the drug candidate in human cells, or a surrogate alternative may be identified through a similar development plan (Bhogal et al., 2008).

\subsection{Economic considerations}

Competition among pharmaceutical companies is intense in the $\mathrm{mAb}$ field, and minimizing discovery and development costs is an important consideration. Because use of the chimpanzee is very expensive, the use of alternatives may provide pharmaceutical companies a competitive edge. As mentioned above, the up-front selection of mAb candidates can take into account the known biological characteristics of typical animal models in order to avoid obvious problems in cross-reactivity and expression of antigens, among other things, in later preclinical studies.

Even if regulatory and ethical considerations were not an issue, the often uncertain benefit that might be derived from the chimpanzee model can make it difficult to justify their use in terms of the cost. The lack of hard data about chimpanzee biology in many respects (Wood, 2006), the less-than-systematical pharmacological history, and the lack of statistical power in chimpanzee studies leave a substantial margin of uncertainty about the predictive quality of this model. Those mAb drugs that have used the chimpanzee in their preclinical studies have shown that the clinical success of such drugs is no better than - and indeed, it appears not to be as good as - that of drugs that did not use them.

Public funding of institutions and investigators that use the chimpanzee in $\mathrm{mAb}$ research also raises significant issues of public policy. A critical and unbiased review of the cost-effectiveness of such research would seem to be required at the time when all areas of scientific research need to accomplish more with fewer funds. Even if such scrutiny were not yet a formal requirement in institutional and funding reviews, both public accountability and fairness within the scientific community would suggest that it be part of funding decisions.

\section{References}

Abi-Rached, L., Moesta, A. K., Rajalingam, R. et al. (2010). Human-specific evolution and adaptation led to major qualitative differences in the variable receptors of human and chimpanzee natural killer cells. PLoS Genet. 6, e1001192.

Alley, S. C., Benjamin, D. C. and Law, C.-L. (2009). Antibodydrug conjugate therapy. In Z. An (ed.), Therapeutic monoclonal antibodies (821-855). Hoboken, NJ, USA: John Wiley \& Sons, Inc.

Almagro, J. C. and Strohl, W. R. (2009). Antibody engineering:
Humanization, affinity maturation, and selection techniques. In Z. An (ed.), Therapeutic monoclonal antibodies (311-334). Hoboken, NJ, USA: John Wiley \& Sons, Inc.

Apoil, P. A., Reid, M. E., Halverson, G. et al. (1997). A human monoclonal anti-D antibody which detects a nonconformation-dependent epitope on the RHD protein by immunoblot. Br. J. Haematol. 98, 365-374.

Arp, J., LeVatte, M., Rowe, J. et al. (1996). A source of glycosylated human T-cell lymphotropic virus type 1 envelope protein: Expression of gp46 by the vaccinia virus/T7 polymerase system. J. Virol. 70, 7349-7359.

Bauer, R. J., Dedrick, R. L., White, M. L. et al. (1999). Population pharmacokinetics and pharmacodynamics of the antiCD11a antibody hu1124 in human subjects with psoriasis. $J$. Pharmacokinet. Biopharm. 27, 397-420.

Bhogal, N., Combes, R. and Balls, M. (2008). Preclinical drug development planning. In S. C. Gad (ed.), Preclinical drug development handbook: Toxicology (1-64). Hoboken, NJ, USA: John Wiley \& Sons, Inc.

Blancher A. and Socha W. W. (1991). Anti-human red cell monoclonal antibodies produced by macaque-mouse heterohybridomas. J. Med. Primatol. 20, 352-356.

Blancher, A., Socha, W. W. and Ruffie, J. (1992). Diversity of human anti-D monoclonal antibodies revealed by reactions with chimpanzee red blood cells. Vox Sang 63, 112-118.

Blewett, E. L., Saliki, J. T. and Eberle, R. (1999). Development of a competitive ELISA for detection of primates infected with monkey B virus (Herpesvirus simiae). J. Virol. Methods 77, 59-67.

Boon, L., Holland, B., Gordon, W. et al. (2002). Development of anti-CD4 mAb hu5a8 for treatment of HIV-1 infection: Preclinical assessment in non-human primates. Toxicology 172, 191-203.

Borke, J. L., Minami, J., Verma, A. et al. (1987). Monoclonal antibodies to human erythrocyte membrane $\mathrm{Ca}^{++}-\mathrm{Mg}^{++}$adenosine triphosphatase pump recognize an epitope in the basolateral membrane of human kidney distal tubule cells. J. Clin. Invest. 80, 1225-1231.

Brekke, O. H. and Sandlie, I. (2003). Therapeutic antibodies for human diseases at the dawn of the twenty-first century. Nat. Rev. Drug Discov. 2, 52-62.

Bukh, J., Thimme, R., Meunier, J.-C. et al. (2008). Previously infected chimpanzees are not consistently protected against reinfection or persistent infection after reexposure to the identical hepatitis C virus strain. J. Virol. 82, 8183-8195.

Chen, Z., Earl, P., Americo, J. et al. (2006a). Chimpanzee/human mAbs to vaccinia virus B5 protein neutralize vaccinia and smallpox viruses and protect mice against vaccinia virus. Proc. Natl. Acad. Sci. USA 103, 1882-1887.

Chen, Z., Moayeri, M., Zhou, Y. H. et al. (2006b). Efficient neutralization of anthrax toxin by chimpanzee monoclonal antibodies against protective antigen. J. Infect. Dis. 193, 625633.

Chimpanzee Sequencing and Analysis Consortium (2005). Initial sequence of the chimpanzee genome and comparison with the human genome. Nature 437, 69-87. 
Crowe, J., Jr., Cheung, P., Wallace, E. et al. (1994). Isolation and characterization of a chimpanzee monoclonal antibody to the $\mathrm{G}$ glycoprotein of human respiratory syncytial virus. Clin . Diagn. Lab. Immunol. 1, 701-706.

Dowell, B. L., Tuck, F. L., Borowitz, M. J. et al. (1984). Phylogenetic distribution of a 24,000 dalton human leukemiaassociated antigen on platelets and kidney cells. Dev. Comp. Immunol. 8, 187-195.

Ehrlich, P. H., Moustafa, Z. A., Justice, J. C. et al. (1988). Human and primate monoclonal antibodies for in vivo therapy. Clin. Chem. 34, 1681-1688.

Emini, E. A., Schleif, W. A., Nunberg, J. H. et al. (1992). Prevention of HIV-1 infection in chimpanzees by gp120 V3 domain-specific monoclonal antibody. Nature 355, 728-730.

Esumi, M., Zhou, Y. H., Tanoue, T. et al. (2002). In vivo and in vitro evidence that cross-reactive antibodies to C-terminus of hypervariable region 1 do not neutralize heterologous hepatitis C virus. Vaccine 20,3095-3103.

FDA (1996). The Center for Drug Evaluation and Research: Application Number NDA 21174. Pharmacology review(s) Part 1. http://www.accessdata.fda.gov/drugsatfda_docs/nda/ 2000/21174_MYLOTARG_pharmr_P1.pdf

FDA (1998). Pharmacology of the infliximab BLA. http://www. fda.gov/downloads/Drugs/DevelopmentApprovalProcess/ HowDrugsareDevelopedandApproved/ApprovalApplications/TherapeuticBiologicApplications/ucm107706.pdf

FDA (1999). Pharmacology review of the infliximab BLA. http:// www.fda.gov/downloads/Drugs/DevelopmentApprovalProcess/HowDrugsareDevelopedandApproved/ApprovalApplications/TherapeuticBiologicApplications/ucm107724.pdf

FDA (2000). The Center for Drug Evaluation and Research: Application number NDA 21174. Pharmacology review(s). Part 2. http://www.accessdata.fda.gov/drugsatfda_docs/nda/ 2000/21174_MYLOTARG_pharmr_P2.pdf

FDA (2003). Efalizumab (Raptiva) approval letter. http://www. fda.gov/downloads/Drugs/DevelopmentApprovalProcess/ HowDrugsareDevelopedandApproved/ApprovalApplications/TherapeuticBiologicApplications/ucm080736.pdf

FDA (2007). Center for Drug Evaluation and Review. Application number 125166: Pharmacology review. http://www.accessdata.fda.gov/drugsatfda_docs/nda/2007/125166s0000_ PharmR.pdf

FDA (2009a). The Center for Drug Evaluation and Research: Application number 125261. Pharmacology review(s). http://www.accessdata.fda.gov/drugsatfda_docs/ nda/2009/125261s000_PharmR.pdf

FDA (2009b). FDA Alert: Information on Tumor Necrosis Factor (TNF) Blockers (marketed as Remicade, Enbrel, Humira, Cimzia, and Simponi). http://www.fda.gov/Drugs/DrugSafety/PostmarketDrugSafetyInformationforPatientsandProviders/ucm109340.htm

FDA (2009c). Raptiva (efalizumab) Feb 2009 - Safety information. http://www.fda.gov/Safety/MedWatch/SafetyInformation/SafetyAlertsforHumanMedicalProducts/ucm149675.htm

FDA (2010a). CDER Therapeutic Biologic Products. http://www.fda.gov/downloads/ForIndustry/UserrFees/ PrescriptionDrugUseerFee/UCM164641.pdf
FDA (2010b). Label and approval history of Orthoclone OKT3. http://www.accessdata.fda.gov/scripts/cder/drugsatfda/index. cfm?fuseaction=Search.Label_ApprovalHistory\#apphist

FDA (2010c). Mylotarg (gemtuzumab ozogamicin): Market Withdrawal. http://www.fda.gov/Safety/MedWatch/ SafetyInformation/SafetyAlertsforHumanMedicalProducts/ ucm 216458.htm

Fishwild, D. M., Hudson, D. V., Deshpande, U. et al. (1999). Differential effects of administration of a human anti-CD4 monoclonal antibody, hm6g, in nonhuman primates. Clin. Immunol. 92, 138-152.

Forns, X., Payette, P. J., Ma, X. et al. (2000). Vaccination of chimpanzees with plasmid DNA encoding the hepatitis $\mathrm{C}$ virus $(\mathrm{HCV})$ envelope $\mathrm{E} 2$ protein modified the infection after challenge with homologous monoclonal HCV. Hepatology 32, 618-625.

Franzoso, G., Hu, P. C., Meloni, G. A. and Barile, M. F. (1993). The immunodominant 90-kilodalton protein is localized on the terminal tip structure of Mycoplasma pneumoniae. Infect. Immun. 61, 1523-1530.

Fridman, E. (2002). Medical primatology. R. D. Nadler (ed.). New York: Francis \& Taylor.

Geijtenbeek, T. B., Koopman, G., van Duijnhoven, G. C. et al. (2001). Rhesus macaque and chimpanzee DC-SIGN act as HIV/SIV gp120 trans-receptors, similar to human DC-SIGN. Immunol. Lett. 79, 101-107.

Genentech (2003). Dermatologic and Ophtalmic Drugs Advisory Committee Meeting, 9 September 2003: Raptiva(TM) (Efalizumab). http://www.fda.gov/ohrms/dockets/ac/03/ briefing/3983B1_01_Genentech-Raptiva.htm

Gibson, C. R., Sandhu, P. and Hanley, W. D. (2009). Monoclonal antibody pharmacokinetics and pharmacodynamics. In Z. An (ed.), Therapeutic monoclonal antibodies (439-460). Hoboken, NJ, USA: John Wiley \& Sons, Inc.

Goncalvez, A. P., Chien, C.-H., Tubthong, K. et al . (2008). Humanized monoclonal antibodies derived from chimpanzee fabs protect against Japanese encephalitis virus in vitro and in vivo. J. Virol. 82, 7009-7021.

Goudsmit, J., Smit, L., Klaver, B. et al. (1987). Induction in chimpanzees of antibodies inhibiting receptor-mediated cell fusion by HIV glycoprotein. Viral Immunol. 1, 225-240.

Hammond, G. L. and Robinson, P. A. (1984). Characterization of a monoclonal antibody to human sex hormone binding globulin. FEBS Lett. 168, 307-312.

Harpprecht, J., Jonker, M., Podzuweit, H. G. et al. (1990). Human monoclonal antibody ha6d3, a candidate for treatment of leukaemia? In vitro reactivity of ha6d3 with leukaemic cells and in vivo applications in a chimpanzee. Br. J. Cancer, Suppl. 10, 44-47.

Hatziioannou, T., Ambrose, Z., Chung, N. P. Y. et al. (2009). A macaque model of HIV-1 infection. Proc. Natl. Acad. Sci. USA 106, 4425-4429.

ICH - International Conference on Harmonization of Technical Requirements for Registration of Pharmaceuticals for Human Use (1997). Preclinical Safety Evaluation of BiotechnologyDerived Pharmaceuticals S6. ICH Harmonized Tripartite Guideline. http://www.ich.org/LOB/media/MEDIA3599.pdf 
Iwarson, S., Tabor, E., Thomas, H. C. et al. (1985). Neutralization of hepatitis B virus infectivity by a murine monoclonal antibody: an experimental study in the chimpanzee. J. Med. Virol. 16, 89-96.

Jansen, J., van der Poll, T., Levi, M. et al. (1995). Inhibition of the release of soluble tumor necrosis factor receptors in experimental endotoxemia by an anti-tumor necrosis factoralpha antibody. J. Clin. Immunol. 15, 45-50.

Jonker, M., Nooij, F. J., den Butter, G. et al. (1988). Side effects and immunogenicity of murine lymphocyte-specific monoclonal antibodies in subhuman primates. Transplantation 45, 677-682.

Jonker, M., Slingerland, W., Treacy, G. et al. (1993). In vivo treatment with a monoclonal chimeric anti-CD4 antibody results in prolonged depletion of circulating $\mathrm{CD} 4^{+}$cells in chimpanzees. Clin. Exp. Immunol.93, 301-307.

Kim, S.-H., Shin, Y. W., Hong, K.-W. et al. (2008). Neutralization of hepatitis B virus (HBV) by human monoclonal antibody against HBV surface antigen (HBsAg) in chimpanzees. Antiviral Research 79, 188-191.

Klotz, F. W., Cohen, S. J., Szarfman, A. et al. (1996). Crossreactive epitope among proteins in Plasmodium falciparum Maurer's clefts and primate leukocytes and platelets. Am. J. Trop. Med. Hyg. 54, 655-659.

Levi, M., ten Cate, H., Bauer, K. A. et al. (1994). Inhibition of endotoxin-induced activation of coagulation and fibrinolysis by pentoxifylline or by a monoclonal anti-tissue factor antibody in chimpanzees. J. Clin. Invest. 93, 114-120.

Levi, M., van Der, P. T., ten Cate, H. et al. (1998). Differential effects of anti-cytokine treatment on bronchoalveolar hemostasis in endotoxemic chimpanzees. Am. J. Respir. Crit. Care Med. 158, 92-98.

Logdberg, L., Kaplan, E., Drelich, M. et al. (1994). Primate antibodies to components of the human immune system. J.Med. Primatol. 23, 285-297.

Loisel, S., Ohresser, M., Pallardy, M. et al. (2007). Relevance, advantages and limitations of animal models used in the development of monoclonal antibodies for cancer treatment. Crit. Rev. Oncol. Hematol. 62, 34-42.

Lu, Y. Q., Liu, J. F., Socha, W. et al. (1987). Polymorphism of glycophorins in nonhuman primate erythrocytes. Biochem. Genet. 25, 477-491.

Maillard, P., Krawczynski, K., Nitkiewicz, J. et al . (2001). Nonenveloped nucleocapsids of hepatitis $\mathrm{C}$ virus in the serum of infected patients. J. Virol. 75, 8240-8250.

McCauley, T. C., Kurth, B. E., Norton, E. J. et al . (2002). Analysis of a human sperm CD52 glycoform in primates: Identification of an animal model for immunocontraceptive vaccine development. Biol. Reprod. 66, 1681-1688.

Men, R., Yamashiro, T., Goncalvez, A. P. et al. (2004). Identification of chimpanzee Fab fragments by repertoire cloning and production of a full-length humanized immunoglobulin G1 antibody that is highly efficient for neutralization of Dengue type 4 virus. $J$ Virol. 78, 4665-4674.

Meunier, J. C., Russell, R. S., Goossens, V. et al. (2008). Isolation and characterization of broadly neutralizing human monoclonal antibodies to the $\mathrm{E} 1$ glycoprotein of hepatitis $\mathrm{C}$ virus. J. Virol. 82, 966-973.
Moller, A., Emling, F., Blohm, D. et al. (1990). Monoclonal antibodies to human tumor necrosis factor alpha: In vitro and in vivo application. Cytokine 2, 162-169.

Nardin, E. H., Nussenzweig, V., Nussenzweig, R. S. et al. (1982). Circumsporozoite proteins of human malaria parasites Plasmodium falciparum and Plasmodium vivax. J. Exp. Med. 156, 20-30.

National Science Board (2002). Public Attitudes Toward Science\&Technology, Scientific Research, Federal Funding of Scientific Research, and Specific Science-Related Issues. http://www.nsf.gov/statistics/seind02/c7/c7s2.htm\#c7s214

Neubauer, R. H., Levy, R., Strand, B. C. and Rabin, H. (1981). Reactivity of monoclonal antibodies against human leucocyte antigens with lymphocytes of non-human primate origin. $J$. Immunogenet. 8, 433-442.

Newman, R., Hariharan, K., Reff, M. et al. (2001). Modification of the Fc region of a primatized IgG antibody to human CD4 retains its ability to modulate CD4 receptors but does not deplete CD4(+) T cells in chimpanzees. Clin. Immunol. 98, 164-174.

Nguyen, D. H. (2006). Loss of siglec expression on T lymphocytes during human evolution. Proc. Natl. Acad. Sci. USA $103,7765-7770$.

Ogata, N., Ostberg, L., Ehrlich, P. H. et al. (1993). Markedly prolonged incubation period of hepatitis $\mathrm{B}$ in a chimpanzee passively immunized with a human monoclonal antibody to the a determinant of hepatitis B surface antigen. Proc. Natl. Acad. Sci. USA 90, 3014-3018.

Palmer, D. K., Morse, H. G. and Jones, C. (1987). Three distinct epitopes of the human MSK20 cell surface antigen are expressed on human and nonhuman primate cells. Mol. Immunol. 24, 865-869.

Parleviet, K. J., Jonker, M., ten Berge, R. J. et al. (1990). AntiCD3 murine monoclonal isotype switch variants tested for toxicity and immunologic monitoring in four chimpanzees. Transplantation 50, 889-892.

Perry, G. H., Yang, F., Marques-Bonet, T. et al. (2008). Copy number variation and evolution in humans and chimpanzees. Genome Research 18, 1698-1710.

Rao, P. E., Olini, G., Kille, J. et al. (1991). OKT3E, an antiCD3 antibody that does not elicit side effects or antiidiotype responses in chimpanzees. Transplantation 52, 691-697.

Sawada, H., Iwasa, S., Nishimura, O. and Kitano, K. (1995). Efficient production of anti-(hepatitis B virus) antibodies and their neutralizing activity in chimpanzees. Appl. Microbiol. Biotechnol. 43, 445-451.

Schofield, D. J., Glamann, J., Emerson, S. U. and Purcell, R. H. (2000). Identification by phage display and characterization of two neutralizing chimpanzee monoclonal antibodies to the hepatitis E virus capsid protein. J. Virol. 74, 5548-5555.

Schofield, D. J., Satterfield, W., Emerson, S. U. and Purcell, R. H. (2002). Four chimpanzee monoclonal antibodies isolated by phage display neutralize hepatitis A virus. Virology 292, 127-136.

Shaw, M. A. (1986). Monoclonal anti-LWab and anti-D reagents recognize a number of different epitopes. Use of red cells of non-human primates. J. Immunogenet. 13, 377-86. 
Socha, W. W., Blancher, A. and Ruffie, J. (1993). Comparative study of human monoclonal anti-D antibodies of IgG and IgM classes in tests with red cells of nonhuman primates. Rev. Fr. Transfus. Hemobiol. 36, 485-497.

Socha, W. W. and Moor-Jankowski, J. (1993). The M-N--V-AB-D blood group system of chimpanzee and other apes: serology and genetics. J. Med. Primatol. 22, 3-6.

Socha, W. W. and Ruffie, J. (1990). Monoclonal antibodies directed against human $\mathrm{Rh}$ antigens in tests with the red cells of nonhuman primates. Rev. Fr. Transfus. Hemobiol. 33, 39-48.

Soto, P. C., Stein, L. L., Hurtado-Ziola, N. et al. (2010). Relative over-reactivity of human versus chimpanzee lymphocytes: Implications for the human diseases associated with immune activation. J. Immunol. 185, 4233-4237.

Strohl, W. R. (2009). Therapeutic monoclonal antibodies: Past, present, and future. In Z. An (ed.), Therapeutic monoclonal antibodies (3-50). Hoboken, NJ, USA: John Wiley \& Sons, Inc.

Takahashi, K., Mishiro, S. and Prince, A. M. (2001). Novel hepatitis B virus strain from a chimpanzee of Central Africa (Pan troglodytes troglodytes) with an unusual antigenicity of the core protein. Intervirology 44, 321-326.

ten Cate, H., Bauer, K. A., Levi, M. et al. (1993). The activation of factor $\mathrm{X}$ and prothrombin by recombinant factor VIIa in vivo is mediated by tissue factor. J. Clin. Invest. 92, 12071212 .

The Pew Research Center for the People \& the Press and The American Association for the Advancement of Science (2009). Public praises science; Scientists fault public, media. http://people-press.org/report/?pageid=1550

Thirkell, D., Myles, A. D. and Taylor-Robinson, D. (1990). A comparison of four major antigens in five human and several animal strains of ureaplasmas. J. Med. Microbiol. 32, 163168.

Toh, B. H., Gibbs, C. J., Jr., Gajdusek, D. C. et al. (1985). The 200- and $150-\mathrm{kDa}$ neurofilament proteins react with IgG autoantibodies from chimpanzees with kuru or CreutzfeldtJakob disease; a $62-\mathrm{kDa}$ neurofilament-associated protein reacts with sera from sheep with natural scrapie. Proc. Natl. Acad. Sci. USA 82, 3894-3896.

Tournamille, C., Blancher, A., Le Van Kim, C. et al. (2004). Sequence, evolution and ligand binding properties of mammalian Duffy antigen/receptor for chemokines. Immunogenetics 55, 682-694.

van der Poll, T., Jansen, J., Levi, M. et al. (1996). Interleukin 10 release during endotoxaemia in chimpanzees: Role of platelet-activating factor and interleukin 6. Scand. J. Immunol.43, 122-125.

Van Meurs, G. J. and Jonker, M. (1986). Production of primate monoclonal antibodies. J. Immunol. Methods 95, 123-128.
VandeBerg, J. L., Zola, S. M., Ely, J. J. and Kennedy, R. C. (2006). Monoclonal antibody testing. J. Med. Primatol. 35, 405-406.

Wang, C. Y., Sawyer, L. S., Murthy, K. K. et al. (1999). Postexposure immunoprophylaxis of primary isolates by an antibody to HIV receptor complex. Proc. Natl. Acad. Sci. USA 96, 10367-10372.

Wang, W., Wang, E. Q. and Balthasar, J. P. (2008). Monoclonal antibody pharmacokinetics and pharmacodynamics. Clin. Pharmacol. Ther. 84, 548-558.

Weinstock, G. M. (2007). ENCODE: More genomic empowerment. Genome Research 17, 667-668.

Winton, E. F., Colenda, K. W., McClure, H. M. et al. (1985). Studies on the use of nonhuman primates to determine the DR status of the human hematopoietic stem cell. Exp. Hematol. 13, 869-73.

Wong, H., Grossman, S. J., Bai, S. A. et al. (2004). The chimpanzee (Pan troglodytes) as a pharmacokinetic model for selection of drug candidates: Model characterization and application. Drug Metab. Dispos. 32, 1359-1369.

Wood, B. (2006). The evolutionary relationships of humans and apes: What we know about human phenotypic specializations. In Arthur M. Sackler (ed.), Colloquia of the National Academy of Sciences: The New Comparative Biology of Human Nature. http://www.nasonline.org/site/ PageServer?pagename=SACKLER_neurosciences_program

World Health Organization (2009). International nonproprietary names. The World Health Organization Drug Information 23:3. http://www.who.int/medicines/publications/druginformation/issues/DrugInfo09vol23_3/en/

Zhang, P., Yu, M. Y., Venable, R. et al. (2006). Neutralization epitope responsible for the hepatitis B virus subtype-specific protection in chimpanzees. Proc. Natl. Acad. Sci. USA 103, 9214-9219.

Zhou, Y. H., Moriyama, M. and Esumi, M. (1999). Multiple sequence-reactive antibodies induced by a single peptide immunization with hypervariable region 1 of hepatitis $\mathrm{C}$ virus. Virology 256, 360-370.

\section{Acknowledgement}

This study was supported in part by The Humane Society of the United States, Washington, D.C., USA.

\section{Correspondence}

Raija H. Bettauer, J.D., M.Sc., LL.M., M.C.L.

Bettauer BioMed Research

Phone: +1 7035360729

e-mail: raija.bettauer@verizon.net 